\title{
PRESENTACIÓN
}

\section{Evaluación de los programas y servicios de salud en México}

L a evaluación permanente de las acciones y resultados alcanzados por los sistemas de salud es una de las principales expresiones de la rectoría del Estado y una de las mejores formas de garantizar que sus objetivos estratégicos se puedan alcanzar. Estas acciones tienen una tendencia creciente en el mundo y actualmente no existe ningún país que pueda organizar seriamente su sistema de salud sin llevarlas a cabo.

No obstante, a pesar de que la evaluación de los sistemas sanitarios se realiza sistemáticamente desde hace por lo menos diez años, la manera de llevarla a cabo ha variado significativamente en el último medio siglo. Como ejemplo, baste mencionar que en el lapso trascurrido de los años cincuenta a los setenta la evaluación de los programas y servicios consistió fundamentalmente en la medición de metas -sobre todo a través de la comparación entre las acciones planeadas y las realizadas- y la medición de la eficiencia alcanzada durante el uso de los recursos. Unas décadas más tarde -probablemente como una de las consecuencias de la difusión que tuvo el modelo de evaluación de la calidad de la atención propuesto por Avedis Donabedian- estas primeras modalidades de evaluación fueron sustituidas paulatinamente por la evaluación simultánea de la estructura, los procesos y los resultados de los programas. Esta manera de evaluar permitió acercarse por primera vez a lo que podría considerarse una evaluación integral de los sistemas sanitarios. Un poco después, al finalizar los años noventa y paralelamente a los movimientos de reforma sanitaria que ocurrieron a escala mundial, surgió el concepto de Evaluación del Desempeño de los Sistemas de Salud, que la Organización Mundial de la Salud formalizó en su Informe Mundial del año 2000. En este caso, como indica su nombre, la evaluación se refiere al desempeño global de los sistemas y es resultado de una combinación de la aplicación de las políticas, la marcha de los programas y los resultados de las acciones de salud.

La evaluación de los sistemas de salud puede realizarse por lo menos en dos niveles. El primero es aquel en el que se encuentran los procesos que el sistema establece en la forma de programas y servicios de salud. El segundo se despliega a escala sistémica, es decir, en materia de grandes políticas. Se denomina Evaluación de Programas y Servicios a la primera modalidad y Evaluación del Desempeño del Sistema de Salud a la segunda. En general, cuando se evalúa el desempeño de los sistemas se evalúan las condiciones generales de salud que se han alcanzado en un país, la brecha sanitaria que existe entre los grupos sociales, el trato que proporciona el sistema a sus usuarios y el grado de eficiencia alcanzado al aplicar los recursos disponibles para atender las necesidades actuales y futuras.

Como puede observarse, la transformación experimentada en el último medio siglo en los modos de evaluar los servicios y sistemas de salud ha consistido, aparentemente, en la sustitución paulatina de la evaluación de la operación de los programas (medida a través del alcance de las metas del programa) por la evaluación del impacto del sistema sobre las condiciones de salud de la población (medida sobre todo a través del nivel de salud alcanzado, las diferencias en las condiciones de los distintos grupos sociales y la equidad en el financiamiento del sistema) como objetivo principal de las evaluaciones.

No obstante, y a pesar de estos avances conceptuales, para los países todavía es muy importante evaluar cada programa y servicio, debido, en primer término, a que la evaluación del desempeño sólo puede ser realizada con los insumos proporcionados por la evaluación de los programas específicos y, en segundo lugar, a que la información sobre cada política y programa es estra- 
tégica para ajustar la marcha de cada uno de ellos al alcance de sus objetivos puntuales. Esto es así porque los servicios de salud son un componente esencial dentro de los sistemas de salud, y representan una síntesis del esfuerzo colectivamente organizado con el fin de prevenir las enfermedades, prolongar la vida y fomentar la salud de la población. México, reconociendo esta importancia, ha establecido dentro del Sistema Nacional de Salud un sistema de evaluación que obliga a cada administración a realizar anualmente la evaluación de los principales programas y servicios de salud.

Para garantizar la objetividad e imparcialidad de los resultados, la normatividad sanitaria establece que la evaluación de los programas y servicios debe estar a cargo de instituciones académicas y de investigación u organismos especializados, de carácter nacional e internacional, que cuenten con amplio reconocimiento y experiencia en la respectiva materia. El propósito de estas evaluaciones es el de identificar los principales retos que enfrenta la marcha de los programas y servicios de salud a nivel nacional, documentar los avances de los programas de salud desplegados en el país y plantear las tareas necesarias para el fortalecimiento de las actividades de salud. Los hallazgos más relevantes de las evaluaciones se sintetizan en una serie de informes técnicos que son usados para llevar a cabo los ajustes pertinentes a cada programa, pero que además se presentan en documentos que se divulgan ampliamente. Es claro que este tipo de evaluación, denominada genéricamente evaluación de programas y servicios, es un proceso significativamente distinto al de la investigación científica. Por esta razón, no es común que los resultados de estas evaluaciones se publiquen en forma de artículos científicos.

Los trabajos que se presentan en este número especial de Salud Pública de México fueron realizados a lo largo de los últimos dos años, y su principal propósito fue generar evidencias de primera mano orientadas a fortalecer el desarrollo de las políticas, programas y servicios de salud a los que cada trabajo se refiere. En general, los trabajos que se presentan documentan evaluaciones hechas ex post, las que se caracterizan por aplicarse a programas que se encuentran plenamente en marcha y permiten ajustar su desarrollo al cumplimiento de sus objetivos finales. En ese sentido, los trabajos coadyuvan a la mejora de la salud de la población al perfeccionar el desarrollo de los programas y servicios a los que se refieren.

No obstante, la característica fundamental de los trabajos es que se trata de documentos diferentes a los informes técnicos presentados como resultado de las evaluaciones convencionales. Conviene recordar en este sentido que la evaluación de programas y servicios tiene como principal objetivo la emisión de un juicio de valor acerca de los alcances de una intervención determinada (acción, programa o servicio) sobre un fenómeno específico (generalmente un grupo de eventos relacionados con la salud). El propósito de las evaluaciones es facilitar el alcance de los objetivos últimos de la intervención -que frecuentemente se refieren a mejorar los indicadores que dan cuenta del fenómeno sanitario-, y por esta razón no siempre se ajustan a los criterios exigidos a las investigaciones propiamente científicas. En el caso de las evaluaciones realizadas a los programas y servicios de salud, por ejemplo, no es común que se hagan explícitos los marcos conceptuales en los que se apoyan, ni que se desarrolle una metodología coherente al marco conceptual y se apliquen únicamente las técnicas e instrumentos que corresponden a cada tipo de investigaciones. Inclusive, es frecuente que se prescinda de hipótesis de trabajo y que únicamente se busque comparar algunos parámetros establecidos convencionalmente con los resultados alcanzados por una intervención dada. El caso de los trabajos que se presentan en este número, empero, es diferente, ya que el rigor teórico y metodológico alcanzado por cada uno de ellos es equiparable a los obtenidos por cualquier investigación científica.

Por esta razón, además de que sus resultados permiten identificar algunos de los más importantes retos del sistema de salud mexicano -al documentar los avances y rezagos de los principales programas de salud pública del país y plantear las tareas necesarias para fortalecerlos- la forma en que cada trabajo fue diseñado y elaborado permite que las conclusiones que presenta puedan ser aplicadas en la formulación, implantación y evaluación de cualquier programa o servicio de salud semejante en el mundo.

En lo que respecta a los objetivos de los trabajos, en general los artículos que aquí se presentan abordan aspectos relativos a la gestión de los programas, la calidad técnica de la atención; la identificación de desigualdades e inequidades en salud; la eficiencia financiera de programas y servicios, y los alcances de la rectoría del Estado en materia sanitaria.

Algunos trabajos tienen como objetivo evaluar la gestión de un programa. Es el caso del que se refiere a las tendencias del fondo de protección contra gastos catastróficos (FPGC); el de separación de funciones en el Sistema de Protección Social en Salud (SPSS); el de evaluación de los gestores de calidad en la Secretaría de Salud, y el del análisis del abasto, surtimiento y gasto de bolsillo en medicamentos en hospitales públicos. La identificación de desigualdades sanitarias fue el propósito del artículo sobre desigualdades actuales y futuras en la frecuencia de la enfermedad renal crónica 
terminal en México, así como del relativo a las consecuencias de la mezcla público privada en el abasto de medicamentos. La equidad en el financiamiento y el impacto económico de los programas se evalúa en los artículos sobre el impacto del Seguro Popular en el gasto catastrófico; abasto, surtimiento y gasto de bolsillo en medicamentos en hospitales públicos, y costo directo de la hemodiálisis en unidades públicas y privadas de la Ciudad de México. Los trabajos que evalúan la calidad de la atención son el del SPSS y la calidad técnica de la atención; el de prevalencia de eventos adversos en pacientes quirúrgicos en instituciones públicas de salud; el de calidad de la atención hospitalaria usando información sobre mortalidad; el que evalúa las características de las unidades de hemodiálisis en México, y el de la enfermedad renal crónica como trazador de la capacidad técnica del sistema de salud.

En materia de evaluación de las políticas públicas en salud se encuentran los artículos sobre las tendencias del FPGC y la organización del abasto de medicamentos en los servicios estatales. Como puede notarse, los propósitos de los trabajos presentados abarcan un amplio abanico que cubre muchos de los objetivos de la evaluación de programas.

Desde el punto de vista metodológico, en la mayor parte de los trabajos se combinan las técnicas cuantitativas y cualitativas, y en algunos casos se hace uso de técnicas complejas de análisis etnográfico. Este fue el caso del estudio sobre las tendencias del FPGC y el de la organización del abasto de medicamentos en los servicios estatales de salud. La revisión de bases de datos especializadas se realizó en los trabajos sobre tendencias del FPGC; el impacto del Seguro Popular en el gasto catastrófico, y el abasto, surtimiento y gasto de bolsillo en medicamentos en hospitales públicos. En los trabajos relativos a la prevalencia de eventos adversos en pacientes quirúrgicos de instituciones públicas, y el SPSS y la calidad técnica de la atención, se revisaron expedientes clínicos. El uso de técnicas etnográficas de recopilación de información -como es el caso de la observación directa y las entrevistas estructurada y semiestructurada- se hizo en los trabajos sobre las tendencias del FPGC; la separación de funciones en el SPSS; la evaluación de los gestores de calidad en la SS y el análisis cualitativo del abasto de medicamentos en México. En los casos de los estudios sobre el impacto del Seguro Popular en el gasto catastrófico y el SPSS y la calidad técnica de la atención se usaron técnicas estadísticas relativamente complejas.

Como puede apreciarse, la mayor parte de los trabajos presentados generaron su propia información primaria, obtenida directamente de los responsables de cada política o programa, sus usuarios (ya sea pacientes o familiares) o a partir de entrevistas realizadas a investigadores o profesionales expertos en el desarrollo de cada programa. La mayor parte de los trabajos validó los instrumentos que aplicó, y usó técnicas estadísticas de complejidad media a superior. Como consecuencia, los análisis que cada trabajo presenta alcanzan una panorámica bastante integral sobre la manera en que cada política y programa se han llevado a cabo en los años estudiados, lo que permite hacer recomendaciones generales y específicas muy puntuales, basadas en las evidencias obtenidas. En todos los casos se busca proporcionar elementos útiles para la redefinición de políticas y acciones específicas en el futuro.

En conjunto, los trabajos que se presentan dan cuenta de la situación que guardan algunos de los principales programas sanitarios desplegados en el país, y representan una muestra de la forma en que se han evaluado en los últimos años. Su lectura cuidadosa puede dar una idea del desempeño reciente de una parte importante del sistema de salud mexicano y proporcionar elementos valiosos para la formulación e implantación de políticas y programas semejantes en otras latitudes. Este es el objetivo de Salud Pública de México al presentar a sus lectores este número especial sobre evaluación de programas y servicios de salud en México.

Francisco Garrido-Latorre. ${ }^{(1)}$ Sergio López-Moreno. ${ }^{(2)}$

(1) Dirección General de Evaluación del Desempeño, Secretaría de Salud. México.

(2) Departamento de Atención a la Salud, División de Ciencias Biológicas y de la Salud, Universidad Autónoma Metropolitana-Xochimilco. México. 